\title{
GROUPOIDS ASSOCIATED WITH ENDOMORPHISMS
}

\author{
VALENTIN DEACONU
}

\begin{abstract}
To a compact Hausdorff space which covers itself, we associate an $r$-discrete locally compact Hausdorff groupoid. Its $\mathbf{C}^{*}$-algebra carries an action of the circle allowing it to be regarded as a crossed product by an endomorphism and as a generalization of the Cuntz algebra $O_{p}$. We consider examples related to coverings of the circle and of a Heisenberg 3-manifold.
\end{abstract}

Given a compact Hausdorff space $X$ and a continuous surjective map $\sigma: X \rightarrow$ $X$, we want to associate to the pair $(X, \sigma)$ a $\mathbf{C}^{*}$-algebra $\mathbf{C}^{*}(X, \sigma)$ which, in the case $\sigma$ is a homeomorphism, coincides with the well-known crossed product $C(X) \times{ }_{\sigma} \mathbf{Z}$.

In this paper we consider the case of a covering $\sigma: X \rightarrow X$ and $\mathbf{C}^{*}(X, \sigma)$ will be defined via a groupoid that generalizes the construction of the Cuntz groupoid $O_{p}$ considered by Renault in [Re]. In that case $X$ is a unilateral sequence space and $\sigma$ is the unilateral shift. We start with some definitions and notation that make sense in greater generality.

Definition. Given a set $X$ and a map $\sigma: X \rightarrow X$, let

$$
\Gamma=\left\{(x, n, y) \in X \times \mathbf{Z} \times X \mid \exists k, l \geq 0, n=l-k, \sigma^{k} x=\sigma^{l} y\right\}
$$

with the set of composable pairs

$$
\Gamma^{(2)}=\{((x, n, y),(w, m, z)) \in \Gamma \times \Gamma \mid w=y\} .
$$

The multiplication and inversion are given by

$$
(x, n, y)(y, m, z)=(x, m+n, z) \quad \text { and } \quad(x, n, y)^{-1}=(y,-n, x) .
$$

The range and source maps are

$$
r(x, n, y)=(x, 0, x), \quad s(x, n, y)=(y, 0, y) .
$$

With these operations $\Gamma$ becomes a groupoid with the unit space identified with $X$. The equivalence relation associated to $\Gamma$ will be denoted by $R$, where

$$
R=\left\{(x, y) \in X \times X \mid \exists k, l \geq 0, \sigma^{k} x=\sigma^{l} y\right\},
$$

and the isotropy group bundle is

$$
I=\left\{(x, n, x) \in X \times \mathbf{Z} \times X \mid \exists k, l \geq 0, n=l-k, \sigma^{k} x=\sigma^{l} x\right\} .
$$

Received by the editors March 25, 1993 and, in revised form, January 21, 1994; originally communicated to the Proceedings of the AMS by Palle E. T. Jorgensen.

1991 Mathematics Subject Classification. Primary 46L05, 46L55, 46L80.

Research supported in part by the National Science Foundation. 
For $k \geq 0$ we consider

$$
R_{k}:=\left\{(x, y) \in X \times X \mid \sigma^{k} x=\sigma^{k} y\right\}
$$

and

$$
R_{\infty}:=\bigcup_{k \geq 0} R_{k}
$$

Note that $R_{\infty} \subset R$.

Theorem 1. Suppose $\sigma: X \rightarrow X$ is a covering. Then $\Gamma$, defined above, carries a topology making it an $r$-discrete locally compact Hausdorff groupoid with the Haar system given by the counting measures.

Proof. Consider on $R_{k}$ the induced topology from $X \times X$. Then $R_{k}$ is a principal $r$-discrete groupoid since $\sigma^{k}$ is a local homeomorphism (see [Kul]). Consider on $R_{\infty}=\bigcup_{k \geq 0} R_{k}$ the inductive limit topology. Since the $R_{k}$ are compact as topological spaces, $R_{\infty}$ is a hyperfinite relation in the terminology of Kumjian (see [Ku2]). Hence $R_{\infty}$ is a principal $r$-discrete groupoid and $X$, identified with the diagonal in $X \times X$, is open in $R_{\infty}$. Let

$$
\tilde{X}=\left\{\left(x_{i}\right) \in \prod_{i \geq 0} X_{i} \mid X_{i}=X, \sigma\left(x_{i+1}\right)=x_{i} \forall i \geq 0\right\}
$$

be the projective limit space and let $\pi_{0}: \tilde{X} \rightarrow X$ the projection onto the first component. With the product topology, $\tilde{X}$ becomes a compact Hausdorff space, and $\pi_{0}$ a continuous open map. The map $\sigma$ induces a homeomorphism $\tilde{\sigma}: \widetilde{X} \rightarrow \widetilde{X}$,

with inverse

$$
\tilde{\sigma}\left(x_{0}, x_{1}, x_{2}, \ldots\right)=\left(\sigma x_{0}, x_{0}, x_{1}, \ldots\right),
$$

$$
\tilde{\sigma}^{-1}\left(x_{0}, x_{1}, x_{2}, \ldots\right)=\left(x_{1}, x_{2}, x_{3}, \ldots\right),
$$

such that $\pi_{0} \circ \tilde{\sigma}=\sigma \circ \pi_{0}($ see $[\mathrm{Br}])$. Let $\widetilde{R}_{\infty}=\left(\pi_{0} \times \pi_{0}\right)^{-1}\left(R_{\infty}\right)$ equipped with the preimage of the inductive limit topology. Then

$$
\widetilde{R}_{\infty}=\left\{\left(\left(x_{i}\right),\left(y_{i}\right)\right) \in \tilde{X} \times \tilde{X} \mid \exists k \geq 0, \sigma^{k} x_{0}=\sigma^{k} y_{0}\right\}
$$

is a principal $r$-discrete groupoid with unit space identified with $\tilde{X}$. The map $\tilde{\sigma}$ induces an automorphism of $\widetilde{R}_{\infty}$ by the formula

$$
\tilde{\sigma}\left(\left(x_{i}\right),\left(y_{i}\right)\right)=\left(\tilde{\sigma}\left(x_{i}\right), \tilde{\sigma}\left(y_{i}\right)\right),
$$

and one can consider the semidirect product $\widetilde{\Gamma}=\widetilde{R}_{\infty} \times_{\tilde{\sigma}} \mathbf{Z}$ (see [Re]). Two elements of $\widetilde{\Gamma}$, written as $\left(\left(x_{i}\right), n,\left(y_{i}\right)\right)$ and $\left(\left(w_{i}\right), m,\left(v_{i}\right)\right)$ with $\left(\left(x_{i}\right),\left(y_{i}\right)\right)$, $\left(\left(w_{i}\right),\left(v_{i}\right)\right) \in \widetilde{R}_{\infty}$, are composable iff $\left(w_{i}\right)=\tilde{\sigma}^{-n}\left(y_{i}\right),\left(v_{i}\right)=\tilde{\sigma}^{-n}\left(z_{i}\right)$ and then the product is given by the formula

$$
\left(\left(x_{i}\right), n,\left(y_{i}\right)\right) \cdot\left(\tilde{\sigma}^{-n}\left(y_{i}\right), m, \tilde{\sigma}^{-n}\left(z_{i}\right)\right)=\left(\left(x_{i}\right), n+m,\left(z_{i}\right)\right) .
$$

The inverse of $\left(\left(x_{i}\right), n,\left(y_{i}\right)\right)$ is $\left(\tilde{\sigma}^{-n}\left(y_{i}\right),-n, \tilde{\sigma}^{-n}\left(x_{i}\right)\right)$. With the induced topology from $\widetilde{R}_{\infty} \times \mathbf{Z}, \widetilde{\Gamma}$ becomes an $r$-discrete locally compact groupoid. Note that $\Gamma=\pi(\widetilde{\Gamma})$, where

$$
\pi: \tilde{X} \times \mathbf{Z} \times \tilde{X} \rightarrow X \times \mathbf{Z} \times X
$$


is given by the formula

$$
\pi\left(\left(x_{i}\right), y,\left(y_{i}\right)\right)=\left(x_{0}, n, \pi_{0}\left(\tilde{\sigma}^{-n}\left(y_{i}\right)\right)\right),
$$

and that $\pi$ is a groupoid homomorphism. With the quotient topology, $\Gamma$ becomes an $r$-discrete locally compact groupoid. It turns out that a basis of open sets for the topology of $\Gamma$ consists of the subsets of the form $U \times\{n\} \times V$, where $n \in \mathbf{Z}, U, V$ are open in $X,\left.\sigma^{n}\right|_{V}: V \rightarrow \tau(U)$ (respectively $\left.\sigma^{-n}\right|_{U}: U \rightarrow$ $\rho(V)$ ) is a homeomorphism for $n \geq 0$ (respectively $n \leq 0$ ), and $\tau, \rho$ are suitable deck transformations for suitable powers of $\sigma$. The equivalence relation $R$ with the trace of this topology was considered also by Vershik and Arzumanian (see [ArVe]). Note that, by construction, $\Gamma$ has a (left) Haar system given by the counting measures.

Definition. If $X$ is compact and $\sigma: X \rightarrow X$ is a covering map, we define $\mathrm{C}^{*}(X, \sigma)$ to be $\mathbf{C}^{*}(\Gamma)$.

Observe that, in the case $\sigma$ is $1-1, \Gamma$ is the transformation group groupoid $X \times \mathbf{Z}$ and $\mathbf{C}^{*}(\Gamma) \simeq C(X) \times{ }_{\sigma} \mathbf{Z}$. But note that when $\sigma$ is not 1-1 (the case in which we are interested), $\mathbf{C}^{*}(\Gamma)$ is more closely allied to a crossed product of $\mathbf{Z}_{+}$on the noncommutative algebra $\mathbf{C}^{*}\left(R_{\infty}\right)$. To see this and in order to study the structure of $\mathbf{C}^{*}(X, \sigma)$, let $c: \Gamma \rightarrow \mathbf{Z}$,

$$
c(x, n, y)=-n \text {. }
$$

Then $c$ is a 1-cocycle, which induces an $S^{1}$-action on $\mathbf{C}^{*}(\Gamma)$ via the formula

$$
(\lambda f)(x, n, y)=\lambda^{-n} f(x, n, y), \quad f \in C_{c}(\Gamma), \lambda \in S^{1},
$$

where $C_{c}(\Gamma)$ are the compactly supported functions on $\Gamma$. The fixed point algebra is $\mathbf{C}^{*}\left(R_{\infty}\right)$, which appears as an inductive limit of $\mathbf{C}^{*}\left(R_{k}\right), k \geq 0$. Kumjian observed in [Kul] that $C^{*}\left(R_{k}\right) \simeq C(X) \times G_{k}$, where $G_{k}$ is the group of deck transformations of the covering $\sigma^{k}: X \rightarrow X$.

Definition. The orbit of $x \in X$ is defined to be

$$
O(x)=\bigcup_{k \geq 0} \sigma^{-k} \sigma^{k} x,
$$

where

$$
\sigma^{-k} y=\left\{z \in X \mid \sigma^{k} z=y\right\}
$$

We say that $\sigma$ minimal if each orbit is dense and that $\sigma$ is essentially free if

$$
\left\{x \in X \mid \forall k, l \geq 0, \sigma^{k} x=\sigma^{l} x \Rightarrow k=l\right\}
$$

is dense in $X$.

Proposition. If $\sigma$ is minimal, then $\mathbf{C}^{*}\left(R_{\infty}\right)$ is simple. Moreover, if $\sigma$ is essentially free, then $\mathrm{C}^{*}(\Gamma)$ is also simple.

Proof. Since $\sigma$ is minimal and

$$
O(x)=\left\{y \in X \mid(x, y) \in R_{\infty}\right\} \subset\{y \in X \mid(x, y) \in R\},
$$

it follows that there are no nontrivial open invariant subsets. If $\sigma$ is also essentially free, then the groupoid $\Gamma$ is essentially principal in the sense of Renault (see Definition II.4.3 of [Re]). Now apply Proposition II.4.6 of [Re], where the ideals of an essentially principal groupoid are characterized. 
The covering map $\sigma$ induces a *-endomorphism of $\mathbf{C}^{*}\left(R_{\infty}\right)$, denoted also by $\sigma$, via the formula

$$
(\sigma f)(x, y)=\frac{1}{p} f(\sigma x, \sigma y), \quad f \in C_{c}\left(R_{\infty}\right),
$$

where $p$ is the index of the covering.

Indeed the fact that this gives an endomorphism follows from these calculations:

$$
(\sigma(f g))(x, y)=\frac{1}{p}(f g)(\sigma x, \sigma y)=\frac{1}{p} \sum_{z \sim \sigma x} f(\sigma x, z) g(z, \sigma y)
$$

and

$$
\begin{aligned}
& ((\sigma f)(\sigma g))(x, y)=\sum_{x \sim w}(\sigma f)(x, w)(\sigma g)(w, y) \\
& =\frac{1}{p^{2}} \sum_{x \sim w} f(\sigma x, \sigma w) g(\sigma w, \sigma y)=\frac{1}{p} \sum_{\sigma x \sim z} f(\sigma x, z) g(z, \sigma y),
\end{aligned}
$$

where $z=\sigma w$ and $x \sim y$ means $(x, y) \in R_{\infty}$.

One can check easily that the induced endomorphism is never unital (unless $\sigma$ is a homeomorphism). However, more important for our purposes, $\sigma$ is induced by a nonunitary isometry and this allows us to represent $\mathbf{C}^{*}(X, \sigma)$ as the crossed product of $\mathrm{C}^{*}\left(R_{\infty}\right)$ by this endomorphism in the sense of Paschke (see [Pa1, Pa2]). In his notation, $B=\mathbf{C}^{*}(\Gamma), A=\mathbf{C}^{*}\left(R_{\infty}\right)$ and the spectral subspace $E_{1}$ is generated by functions supported on $\{(x, n, y) \in \Gamma \mid n=-1\}$. Let

Then $v \in E_{1}$,

$$
v(x, n, y)= \begin{cases}1 / \sqrt{p} & \text { if } n=-1 \text { and } y=\sigma x \\ 0 & \text { otherwise }\end{cases}
$$

$$
\left(v^{*} v\right)(x, n, y)= \begin{cases}1 & \text { if } x=y \text { and } n=0 \\ 0 & \text { otherwise }\end{cases}
$$

Thus $v$ is an isometry in $B$ and

$$
\left(v v^{*}\right)(x, n, y)= \begin{cases}1 / p & \text { if } \sigma x=\sigma y \text { and } n=0 \\ 0 & \text { otherwise }\end{cases}
$$

is a projection in $A$, different from 1. Moreover, $\sigma f=v f v^{*}$. These calculations lead to

Theorem 2. Suppose $\sigma: X \rightarrow X$ is minimal. Then we have an exact sequence

$$
\begin{aligned}
\cdots \rightarrow K_{0}\left(\mathbf{C}^{*}\left(R_{\infty}\right)\right) \stackrel{\sigma_{*}-\mathrm{id}}{\longrightarrow} K_{0}\left(\mathbf{C}^{*}\left(R_{\infty}\right)\right) \stackrel{i_{*}}{\longrightarrow} K_{0}\left(\mathbf{C}^{*}(X, \sigma)\right) \rightarrow \\
\quad \rightarrow K_{1}\left(\mathbf{C}^{*}\left(R_{\infty}\right)\right) \stackrel{\sigma_{*}-\mathrm{id}}{\longrightarrow} K_{1}\left(\mathbf{C}^{*}\left(R_{\infty}\right)\right) \stackrel{i_{*}}{\rightarrow} K_{1}\left(\mathbf{C}^{*}(X, \sigma)\right) \rightarrow \cdots
\end{aligned}
$$

where $i: \mathbf{C}^{*}\left(R_{\infty}\right) \hookrightarrow \mathbf{C}^{*}(X, \sigma)$ is the inclusion map and id is the identity on K-theory.

Proof. Using the fact that $\sigma f=v f v^{*}$, it follows that $\sigma\left(\mathbf{C}^{*}\left(R_{\infty}\right)\right)$ is a corner in $C^{*}\left(R_{\infty}\right)$. Since $C^{*}\left(R_{\infty}\right)$ is simple, it is a full corner and we may apply Theorem 4.1 of $[\mathrm{Pa} 2]$. Notice that

$$
\sigma: \mathbf{C}^{*}\left(R_{\infty}\right) \rightarrow \sigma\left(\mathbf{C}^{*}\left(R_{\infty}\right)\right)
$$


is an isomorphism, therefore $\sigma_{*}$ is an isomorphism at the level of $K$ - theory.

We turn now to some examples that illustrate the applicability and the limitations of our analysis.

Example 1. Let $X=\prod_{i \geq 0} X_{i}$, where $X_{i}=\{1,2, \ldots, p\}$ for each $i \geq 0$, with product topology, and let $\sigma: X \rightarrow X$ be the unilateral shift,

$$
\sigma\left(x_{0}, x_{1}, \ldots\right)=\left(x_{1}, x_{2}, \ldots\right) \text {. }
$$

Then $\sigma: X \rightarrow X$ is a $p$-fold covering. In this case

$$
\mathbf{C}^{*}\left(R_{k}\right) \simeq M_{p^{k}}(C(X)),
$$

$R_{\infty}$ is the Glimm equivalence relation,

$$
\mathrm{C}^{*}\left(R_{\infty}\right) \simeq U H F\left(p^{\infty}\right),
$$

and $C^{*}(X, \sigma)$ is the Cuntz algebra $O_{p}$. Note that $\sigma$ is essentially free since the points in $X$ with trivial isotropy are the nonperiodic sequences, which are dense in $X$. The fact that $U H F\left(p^{\infty}\right)$ and $O_{p}$ are simple is well-known.

Example 2. Let $A=(A(i, j))$ be a $p \times p$ matrix of 0 's and 1's, let $X$ be as in example 1 and let

$$
X_{A}=\left\{\left(x_{i}\right) \in X \mid A\left(x_{i}, x_{i+1}\right)=1\right\} .
$$

Define $\sigma_{A}: X_{A} \rightarrow X_{A}$ to be the restriction of $\sigma$ to $X_{A}$. For simplicity, we require that

$$
\sum_{i=1}^{p} A(i, j)=q \quad \forall j
$$

for some $q \geq 2$, so that $\sigma_{A}$ is a $q$-fold covering. (Without this condition, we would consider different $q$ 's for different pieces of $\left.X_{A}\right)$. Then

$$
\mathrm{C}^{*}\left(R_{k}\right) \simeq M_{q^{k}}\left(C\left(X_{A}\right)\right),
$$

$\mathrm{C}^{*}\left(R_{\infty}\right)$ is an $A F$-algebra, and $\mathrm{C}^{*}\left(X_{A}, \sigma_{A}\right)$ is the Cuntz-Krieger algebra $O_{A}$. If the matrix $A$ is aperiodic, then $\sigma_{A}$ is minimal and essentially free, so that $\mathrm{C}^{*}\left(R_{\infty}\right)$ and $O_{A}$ are simple (again a well-known result).

Example 3. Let $X=\mathbf{T}, \sigma: \mathbf{T} \rightarrow \mathbf{T}, \sigma(x)=x^{p}, p \geq 2$. In this case $\mathbf{C}^{*}\left(R_{k}\right) \simeq$ $M_{p^{k}}(C(\mathrm{~T}))$ (see $\left.[\mathrm{Ku} 1]\right)$, and $\mathrm{C}^{*}\left(R_{\infty}\right)$ is the Bunce-Deddens algebra of type $p^{\infty}$. It is known that

$$
K_{0}\left(\mathbf{C}^{*}\left(R_{\infty}\right)\right) \simeq \mathbf{Z}[1 / p], K_{1}\left(\mathbf{C}^{*}\left(R_{\infty}\right)\right) \simeq \mathbf{Z}
$$

(see [B1]) and it is easy to see that $\sigma$ is minimal. Thus one may apply the exact sequence in Theorem 2 to compute the $K$-theory of $\mathbf{C}^{*}(\mathbf{T}, \sigma)$. In order to determine $\sigma_{*}$, observe that $\sigma\left(\mathbf{C}^{*}\left(R_{k}\right)\right) \subset \mathbf{C}^{*}\left(R_{k+1}\right)$ and it will be enough to know the maps at the level of $K$-theory induced by

$$
\mathbf{C}^{*}\left(R_{0}\right)=C(\mathbf{T}) \stackrel{\sigma}{\longrightarrow} M_{p}(C(\mathbf{T}))=\mathbf{C}^{*}\left(R_{1}\right) .
$$

Borrowing notation from [Kul], $l^{2}(\sigma)$ is a $C(\mathbf{T})$-Hilbert module via the inner product

$$
(f \mid h)(x)=\sum_{\sigma y=x} \bar{f}(y) h(y), \quad f, h \in C(\mathbf{T})
$$


with basis $g_{j}(x)=x^{j} / \sqrt{p}, j=0,1, \ldots, p-1$. Furthermore, $\mathbf{C}^{*}\left(R_{1}\right) \simeq$ $\mathscr{K}\left(l^{2}(\sigma)\right)$. Since $f \in C\left(R_{1}\right)$ may be viewed as an element of $\mathscr{K}\left(l^{2}(\sigma)\right)$ via the formula

$$
(f g)(x)=\sum_{y} f(x, y) g(y), g \in l^{2}(\sigma),
$$

one can identify $f$ with the matrix $\left(f_{l j}\right)$, where $f_{l j}=\left(g_{l} \mid f g_{j}\right)$. So, letting $x=e(t)=\exp (2 \pi i t)$, we see that

$$
\begin{aligned}
f_{l j}(e(t))=\frac{1}{p} \sum_{k, m=0}^{p-1} e((t(j-l)+k(j-l)+m j) / p) f(e((t+k) / p), & e((t+m+k) / p)) .
\end{aligned}
$$

Following these identifications, the projection $\sigma(1)=v v^{*}$ corresponds to $e_{11} \otimes 1$ in $M_{p}(C(\mathbf{T}))$, so that

$$
\mathbf{Z}=K_{0}(C(\mathbf{T})) \stackrel{\sigma_{*}}{\longrightarrow} K_{0}\left(M_{p}(C(\mathbf{T}))\right)=\mathbf{Z}
$$

is the identity map. If we denote by $u$ the unitary

$$
u(x, n, y)= \begin{cases}x & \text { if } y=x \text { and } n=0 \\ 0 & \text { otherwise }\end{cases}
$$

which generates $C(\mathbf{T})$, then $v u v^{*}$ corresponds in $M_{p}(C(\mathbf{T}))$ to $e_{11} \otimes u$ so that

$$
\mathbf{Z}=K_{1}(C(\mathbf{T})) \stackrel{\sigma_{*}}{\longrightarrow} K_{1}\left(M_{p}(C(\mathbf{T}))\right)=\mathbf{Z}
$$

is again the identity map. Therefore $\sigma_{*}: K_{0}\left(\mathbf{C}^{*}\left(R_{\infty}\right)\right) \rightarrow K_{0}\left(\mathbf{C}^{*}\left(R_{\infty}\right)\right)$ is the multiplication by $1 / p$, and $\sigma_{*}: K_{1}\left(\mathbf{C}^{*}\left(R_{\infty}\right)\right) \rightarrow K_{1}\left(\mathbf{C}^{*}\left(R_{\infty}\right)\right)$ is the identity map. We get the exact sequences

$$
0 \rightarrow \mathbf{Z}_{p-1} \rightarrow K_{0}\left(\mathbf{C}^{*}(\mathbf{T}, \sigma)\right) \rightarrow \mathbf{Z} \rightarrow 0
$$

and

$$
0 \rightarrow \mathbf{Z} \rightarrow K_{1}\left(\mathbf{C}^{*}(\mathbf{T}, \sigma)\right) \rightarrow 0,
$$

from which it follows that

$$
K_{0}\left(\mathbf{C}^{*}(\mathbf{T}, \sigma)\right) \simeq \mathbf{Z} \oplus \mathbf{Z}_{p-1} \quad \text { and } \quad K_{1}\left(\mathbf{C}^{*}(\mathbf{T}, \sigma)\right) \simeq \mathbf{Z} .
$$

The isotropy groups

$$
I_{x}=\left\{n \in \mathbf{Z} \mid x^{p^{k}}=x^{p^{l}}, l-k=n\right\}
$$

are trivial for a dense set of $x$ in $\mathbf{T}$, therefore $\sigma$ is essentially free and $\mathbf{C}^{*}(\mathbf{T}, \sigma)$ is a simple $\mathbf{C}^{*}$-algebra. Since the corresponding isometry $v$ is nonunitary, it follows that $\mathbf{C}^{*}(\mathbf{T}, \sigma)$ is infinite.

One can consider coverings of $\mathbf{T}^{q}$ for $q \geq 2$ to get other simple infinite C*$^{*}$-algebras.

Example 4. Consider the real Heisenberg group of dimension 3, $N$. We write the elements of $N$ as triples $(x, y, z) \in \mathbf{R}^{3}$ with multiplication

$$
(x, y, z)\left(x^{\prime}, y^{\prime}, z^{\prime}\right)=\left(x+x^{\prime}, y+y^{\prime}, z+z^{\prime}+x y^{\prime}\right) \text {. }
$$

Consider also the discrete Heisenberg group

$$
L=\{(x, y, z) \in N \mid x, y, z \in \mathbf{Z}\},
$$


and let $X=N / L$. This is a compact 3-manifold (a circle bundle over $\mathbf{T}^{2}$ ). The elements of $X$ will be written as $[x, y, z], x, y, z \in[0,1)$. Let

$$
s: N \rightarrow N, \quad s(x, y, z)=(p x, q y, p q z),
$$

where $p, q \geq 2$ are integers. Since $s(L) \subset L$, we get a map

$$
\sigma: X \rightarrow X, \quad \sigma[x, y, z]=[p x, q y, p q z],
$$

which is a $p^{2} q^{2}$-fold covering (see [Sh]). Invoking the methods of Kumjian [Ku1] again, we observe that a basis for the $C(X)$-Hilbert module $l^{2}(\sigma)$ is given by

$$
\begin{aligned}
& g_{l, m, n}[x, y, z]=\frac{1}{p q} e(l x+m y+n z), \quad l=0, \ldots, p-1, \\
& m=0, \ldots, q-1 \text {, } \\
& n=0, \ldots, p q-1 \text {, }
\end{aligned}
$$

where $e(a)=\exp (2 \pi i a)$. This allows us to identify $\mathbf{C}^{*}\left(R_{1}\right)$ with $M_{p^{2} q^{2}}(C(X))$. Therefore $\mathrm{C}^{*}\left(R_{\infty}\right)$ will be an inductive limit of matrix algebras over $C(X)$.

Observe that $\sigma$ is minimal and essentially free, so that $\mathbf{C}^{*}\left(R_{\infty}\right)$ and $\mathbf{C}^{*}(X, \sigma)$ are simple. It is known that the computation of $K_{*}(C(X))$ may be reduced to the computation of $K_{*}\left(\mathrm{C}^{*}(L)\right)$, which is done by Anderson and Paschke (see [AnPa]). The computation of the $K$-theory of $\mathbf{C}^{*}(X, \sigma)$ using Theorem 2 can proceed, at least in principle. However at the moment, the details appear quite complicated.

Remark. If $\sigma: X \rightarrow X$ is a branched covering like $z \rightarrow z^{p}$ on the closed unit disc $\mathbf{D}^{2}$, one can define a locally compact topology on the groupoid $\Gamma$ using the procedure described above. However, it will no longer be $r$-discrete. The reason is that the family of measures

$$
\lambda^{(0,0)}(f)=2 f(0,0), \quad \lambda^{(x, x)}(f)=f(x, x)+f(x,-x), \quad x \neq 0,
$$

is a Haar system for

$$
R_{1}=\left\{(x, y) \in \mathbf{D}^{2} \times \mathbf{D}^{2} \mid x^{2}=y^{2}\right\}
$$

which does not consist of counting measures (see [Re]).

Notice that $\mathbf{D}^{2} \backslash\{0\}$ is an open invariant subset of the unit space $\mathbf{D}^{2}$. Groupoids associated with branched coverings will be discussed elsewhere.

\section{ACKNOWLEDGMENTS}

The author would like to express his gratitude to Professor P. Muhly for pointing out the problem and for constant support during the writing of this paper, and to Professors J. Spielberg and I. Putnam for pointing out some errors in earlier drafts.

\section{REFERENCES}

[AnPa] J. Anderson and W. L. Paschke, The rotation algebra, Houston J. Math. 15 (1989), 1-26.

[ArVe] V. A. Arzumanian and A. M. Vershik, Star algebras associated with endomorphisms, Operator Algebras and Group Representations, vol. I, Pitman, 1984, pp. 17-27.

[B1] B. Blackadar, K-theory for operator algebras, Springer-Verlag, 1986. 
[Br] J. R. Brown, Ergodic theory and topological dynamics, Academic Press, 1976.

[Ku1] A. Kumjian, Preliminary algebras arising from local homeomorphisms, Math. Scand. 52 (1983), 269-278.

[Ku2] _ On $C^{*}$-diagonals, Canad. J. Math. 38 (1986), 969-1008.

[Mu] P. Muhly, Coordinates in operator algebras (to appear).

[Pa1] W. L. Paschke, The crossed product of a $C^{*}$-algebra by an endomorphism, Proc. Amer. Math. Soc. 80 (1980), 113-118.

[Pa2] $-K$-theory for actions of the circle group on $C^{*}$-algebras, J. Operator Theory 6 (1981), 125-133.

[Re] J. Renault, $A$ groupoid approach to $C^{*}$-algebras, Lecture Notes in Math., vol. 793, SpringerVerlag, 1980.

[Sh] M. Shub, Endomorphisms of compact differentiable manifolds, Amer. J. Math. 91 (1969), 175-199.

Department of Mathematics, University of lowa, Iowa City, Iowa 52242

E-mail address: vdeaconu@oak.math.uiowa.edu 